\title{
Comparación de la estimación de la productividad del proceso de soldadura eléctrica por los métodos de simulación de Monte Carlo e Hipercubo Latino
}

\author{
José L. F. Martins, Miguel L. R. Ferreira, Juan M. Pardal y Cássia A. R. Morano \\ Universidade Federal Fluminense, Escola de Engenharia, Rua Passo da Pátria 156, São \\ Domingos Niterói, Rio de Janeiro-Brasil (e-mail: jlfmtem@vm.uff.br, miguelluiz@lamis.uff.br, \\ juanpardal@vm.uff.br, carm@lamis.uff.br)
}

Recibido Oct. 06, 2011; Aceptado Nov. 22, 2011; Versión final recibida Ene. 11, 2012

\begin{abstract}
Resumen
Se evalúan los métodos de simulación de Monte Carlo e Hipercubo Latino para estimar la productividad del proceso de soldadura eléctrica por electrodo revestido (shielded metal arc welding) en las obras de montaje de tuberías industriales considerando pequeñas muestras. EI estudio se realizó mediante el análisis de una muestra recogida en el ámbito de las obras en la Refinería Duque de Caxias, Estado de Río de Janeiro en Brasil. Se utilizó el programa ControlTub 5.3 para la adquisición de los datos y el programa de MathWorks para la simulación, Para el análisis de los datos se comparó los resultados obtenidos mediante los métodos estudiados. Posteriormente, se evaluaron las similitudes y diferencias entre estos métodos en la estimación de la productividad del proceso. Los resultados demostraron la viabilidad de aplicar los métodos de simulación de Monte Carlo e Hipercubo Latino en la estimación de la productividad en el proceso de soldadura de tuberías industriales.
\end{abstract}

\section{Comparison in estimating productivity of Shielded Metal Arc Welding process through Monte Carlo and Latin Hypercube Sampling Simulation Methods}

\begin{abstract}
The ability of Monte Carlo simulation and Latin Hypercube Sampling methods in estimating the productivity of the Shielded Metal Arc Welding (SMAW) applied in works of industrial piping assembly based on small samples is evaluated. The study was conducted by analyzing a sample collected from assembly operations done at refinery Duque de Caxias, Rio de Janeiro in Brazil. The ControlTub 5.3 software was used for data acquisition and MathWorks in the simulation step. For data analysis the results were compared using the proposed simulation methods. Then the similarities and differences between the methods to estimate the process productivity in analysis were evaluated. The results demonstrated the feasibility of applying the methods of Monte Carlo Simulation and Latin Hypercube Sampling in estimating productivity considering a welding process of industrial pipes.
\end{abstract}




\section{INTRODUCCION}

El objetivo de este estudio es comparar la aplicabilidad de los métodos de Monte Carlo (MMC) e Hipercubo Latino, o "Latin Hypercube Sampling" (LHS), en la estimación de la productividad en la soldadura de uniones soldadas, a través de la simulación en muestras pequeñas. La muestra de referencia se refiere a la base de datos históricos recogidos por Gioia y Silva Junior (2007). A partir de esta muestra de referencia se tomaron muestras aleatorias, considerando cada una de ellas como si los datos restantes del conjunto muestral fuesen desconocidos. A partir de cada muestra pequeña real son generados datos virtuales por simulaciones de MMC e LHS como la generatriz de la función de densidad de probabilidad (FDP) y la función de probabilidad acumulada (FPA). A continuación, se comparan las curvas generadas con la curva equivalente de la muestra de referencia. En el análisis de los resultados se comparan los dos métodos de simulación propuestos. Posteriormente, se evalúan las similitudes y diferencias entre ellos en la estimación de la productividad del proceso de soldadura eléctrica de electrodo revestido. Este estudio es basado en los trabajos realizados por Martins (2011). EI MMC e LHS presentan características específicas de aplicación, siendo realizada una comparación entre los métodos, evaluando sus ventajas y desventajas, como bien las diferencias en los resultados presentados por estos métodos de simulación. Una vez verificada la aplicabilidad de cada uno de estos métodos, existe la posibilidad de comparación de estas herramientas para monitorear la productividad en la soldadura, en la preparación de estimaciones de costos y en los plazos durante en la construcción y montaje de tuberías industriales.

En los métodos y procesos utilizados en la industria de la construcción es requerida la introducción de los recursos humanos, provisión de recursos financieros y equipamientos, lo que resulta en un producto. Tradicionalmente, la productividad se define por la relación entre los recursos humanos y el producto resultante. Según Diekmann y Heinz (2001), la productividad es la relación de Horas-hombre [Hh] utilizadas en el proceso de conversión constructiva con la cantidad de productos obtenidos. El termino Horas-hombre es definido por la actividad del trabajador en una hora de trabajo. Esta relación es comúnmente utilizada por la industria brasileira. En el caso de la soldadura, la productividad es, en general, la cantidad de metal de soldadura depositado en relación a la cantidad de recursos consumidos en la soldadura. En el proceso de soldadura eléctrica de electrodo revestido, la productividad es la relación entre el volumen $\left[\mathrm{cm}^{3}\right]$ o masa $[\mathrm{kg}]$ depositados y las $[\mathrm{Hh}]$ Horas-hombre utilizadas en el proceso de soldadura. Por lo tanto, la unidad utilizada es $\left[\mathrm{cm}^{3} / \mathrm{Hh}\right]$ o [kg / Hh]. Se verifica que, dimensionalmente la medida de productividad en el proceso de soldadura es representada por la inversa de la definición de Diekmann e Heinz (2001), ya que en este caso es interesante evaluar la medida de la productividad en lugar de la improductividad, como citado por los autores.

Para el monitoreo de la productividad en la soldadura se mencionan en la literatura varios indicadores. Entre ellos, hay quienes consideran sólo el metal depositado con el arco abierto teniendo en cuenta el tiempo de ejecución total de la junta, ya que éstos se utilizan comúnmente en la industria, como se indica en el "Programa de Mobilização da Indústria de Petróleo e Gas Natural", PROMINP (2010). Este programa fue escrito con la participación de la industria de la construcción y montaje de Brasil, que estableció indicadores de padrones de productividad para la soldadura. Además, hay otros importantes estudios sobre las tasas de productividad en la soldadura, que se ocupan de la importancia de tal medida. El más destacado es relativo a las consideraciones de Page y Nation (1967), que realizaron una aproximación global sobre el uso de Horas-hombre en diversos entornos y situaciones de soldadura. La American Welding Society, AWS (2002), establece ocho tipos de medidas generales de la productividad y, por otra parte, Brito y Paranhos (2005) analizaron la dependencia del proceso de soldadura en la productividad, en los diversos métodos y procesos utilizados en la industria.

En el caso de indicadores que tengan en cuenta el tiempo de ejecución total de la junta que se refieren, en su mayoría, al volumen de la soldadura por la cantidad de Horas-hombre [Hh] que se emplean en la operación de soldadura. En relación a la mano de obra se encuentran las siguientes situaciones: únicamente la cantidad de soldadores [Hh]; cantidad de [Hh] de los soldadores y ayudantes, además de la cantidad de [Hh] de los soldadores, ayudantes y 
supervisores de soldaduras de nivel de escolaridad bajo, que son normalmente intitulados de encargado de soldadura por la industria brasileira.

Los indicadores se sustentan en los datos históricos de Gioia Junior y Silva (2009), donde se consta la productividad de cada registro de soldador en un determinado número de días trabajados, expresado en junta equivalente [Juequi] y convertido para $\left[\mathrm{cm}^{3} / \mathrm{Hh}\right.$ ]. Las medidas de tiempo para evaluar la equivalencia de las medidas fueron obtenidas en campo teniendo en cuenta la situación real de la obra en que, por ejemplo, el soldador debe dejar de soldar por falta de material, para ajustar la máquina de soldar, para tomar un café y/o otros aspectos vinculados al ambiente de trabajo. Por otra parte, la productividad es medida considerando que el inicio del cronometraje del tiempo debe coincidir con el inicio de la soldadura de la junta y el término del mismo debe ocurrir en la limpieza final, posterior al pase final de soldadura.

\section{MONTE CARLO (MMC) e HIPERCUBO LATINO (LHS)}

La simulación de Monte Carlo (MMC) se basa en la generación de una serie de números pseudo aleatorios previamente establecidos con sus valores distribuidos de acuerdo a una función con parámetros identificados en la muestra a ser analizada evaluando su cumplimiento en relación con la distribución de los elementos de la muestra. La función generatriz a ser utilizada como FPD debe presentar una forma semejante a la distribución de los elementos de la muestra para las clases a ser calculadas en condiciones predeterminadas. La cantidad de números pseudo aleatorios que se generarán para la simulación se determinan de acuerdo con las recomendaciones formuladas por Cochran (1954), Roberts (2001), Flanagan (1993) y Morano (2003). Así, la simulación se lleva a cabo por el tamaño de la muestra, las clases y la cantidad del conjunto de números pseudo aleatorios. Luego, se realiza la verificación de la distribución de los números generados en relación a la distribución inicial, por la prueba de adherencia Chi cuadrado y por comparación de la máxima verosimilitud de la simulación en relación a la muestra. Con la condición de aprobación de la prueba de adherencia, se valida la simulación y se realiza la construcción de las figuras con los resultados obtenidos.

El método de Hipercubo Latino (LHS) es similar al MMC con la diferencia básica que las clases son tratadas como estratos y los números pseudo aleatorios se distribuyen en una distribución proporcional a los elementos de cada muestra entre los estratos establecidos. Pilger (2005) realizó un enfoque profundo en los métodos de simulación y el aumento de su eficiencia, presentando una propuesta de sustituir el método de Monte Carlo por la técnica de muestreo Hipercubo Latino (LHS) que refleja una mayor precisión y velocidad que el método proporciona para cualquier modelo de incertidumbre. Maschio (2009) mostró la aplicación de la técnica de LHS en la integración del ajuste histórico de producción en el análisis de incertidumbres con vista a modelos más confiables para la previsión de producción de petróleo y gas. La función generatriz a ser utilizada como FDP es generada a partir de la unión de los puntos medios de cada estrato y debe presentar forma semejante a de la distribución de los elementos de la muestra por los estratos a ser calculados en condiciones predeterminadas. La cantidad de números pseudo aleatorios a ser generados para la simulación son establecidos de manera semejante a la descripta en la MMC. De esta manera, la simulación es ejecutada, a partir de la muestra, con los estratos y la cantidad de números pseudo aleatorios definidos proporcionalmente a cada estrato de la muestra. A continuación es realizada la verificación de la distribución de los números pseudo aleatorios en relación a la proporción de la distribución inicial estratificada, por la prueba de adherencia Chi cuadrado y por comparación de la máxima verosimilitud de la simulación en relación a la pequeña muestra. Con la condición de la prueba de adherencia aprobada, se valida la simulación y se realiza la construcción de las figuras con los resultados obtenidos.

\section{PROCEDIMIENTO EXPERIMENTAL}

El procedimiento del experimento se llevó a cabo teniendo en cuenta el plan de muestreo que se define como la relación de los procedimientos para la aplicación de técnicas de muestreo para recoger una muestra representativa. En este contexto, se presentan los siguientes pasos secuenciales: 1) la definición de la población en que desea hacer la estimación; 2) definición de la 
técnica muestral y su justificativa; 3) establecimiento de las técnicas de colecta aleatoria y almacenamiento de datos; 4) adopción de un nivel de confianza; 5) establecimiento de el tamaño de la muestra por muestra preliminar; 6) análisis de los datos que constituyen la muestra; 7) distribución de los datos en clases en el MMC y en estratos en el LHS; 8) adopción de la FDP adecuada por la evaluación de la máxima verosimilitud en la muestra; simulación por el método de Monte Carlo o Hipercubo Latino; 9) pruebas de adherencia por la prueba de Chi-cuadrado y por la comparación de la máxima verosimilitud de la muestra y de la simulación; 10) prueba de hipótesis; 11) retorno al inicio o establecimiento de la FPA y construcción de las figuras de los resultados.

Para el tratamiento de los datos de la muestra, se deben completar los siguientes pasos: 1) la comprobación de que si la muestra recogida es adecuada para el nivel de importancia establecido. Siendo así, se agrupan los datos en una tabla con intervalos de clases o estratos, para la construcción del histograma de frecuencias. De lo contrario, debe ser efectuada una colecta hasta que haya una mayor compatibilidad con el tamaño de la muestra y la importancia del intervalo predeterminado. 2) La evaluación de que la distribución de FDP es una variable aleatoria que representa los datos de una muestra mejor organizada, de conformidad con el paso 1. Para esta evaluación, la prueba de máxima verosimilitud es muy adecuada en función de la capacidad de comparar la función que mejor se adapte a las características de la muestra que es objeto de análisis. 3) Después de la aplicación de los pasos 1 y 2 , se adoptará algún o ambos métodos de simulación descriptos, que deben ejecutar la simulación con la generación de números pseudo aleatorios correspondiente a cada método basado en la distribución definida en el ítem 2, teniendo en cuenta los rangos establecidos en el ítem 1. 4) Comprobar si el número de simulaciones es satisfactoria, de lo contrario, el ítem 3 deberá repetirse hasta que el número ideal de simulaciones sea alcanzado. La validación por el método de Monte Carlo se realiza mediante la prueba de Chicuadrado para evaluar el grado de adherencia de los datos de ejemplo en comparación con datos simulados, o mediante la comparación de la máxima verosimilitud. Para el método de Hipercubo Latino se dividen los atributos en estratos y se realizan sorteos equivalentes al porcentaje asignado a cada estrato, además el cumplimiento de la evaluación se realiza de manera similar a la utilizada en el MMC. 5) A partir de las simulaciones de la etapa 4, se construye la función de probabilidad acumulada (FPA), que mediante el análisis y evaluación de la productividad del proceso se lleva a cabo una comparación de los métodos.

\section{Descripción de la Base de Datos}

Como se mencionó anteriormente, este trabajo utilizó la base de datos de Gioia e Silva Junior (2009), además de las pequeñas muestras establecidas por Constancio (2009). Se consideraron como muestras de referencia los datos colectados de al menos 10 días efectivos de trabajo. Este criterio se adoptó sobre la base de la práctica operativa, percibiéndose que un soldador con diez días de actividad efectivos se encuentra en buenas condiciones de productividad (Constancio, 2009). Los elementos de la base de datos se obtuvieron de las obras en la Refinería Duque de Caxias (REDUC), en Río de Janeiro, Brasil, cuyas medidas fueron realizadas por el contratista, verificados y validados por el ente fiscalizador de la obra con el auxilio del software ControlTub 5.3.

Las muestras colectadas son presentadas en la forma de valores medios diarios de los indicadores de productividad para cada actividad en el campo de cada soldador. Los elementos de las muestras se introdujeron en el software con cada soldador representado por un sello o identificación alfanumérica intrínseca. Estos indicadores son tratados y presentados en términos del volumen de soldadura depositado en la junta por las horas hombre $\left[\mathrm{cm}^{3} / \mathrm{Hh}\right]$, en el montaje de la tubería. La muestra colectada consiste de 160 elementos, la cual se denomina 160el. Luego, pequeñas muestras se toman al azar de la muestra de referencia, con 20, 15 y 10 elementos cada una, denominándolas de $20 \mathrm{el}, 15 \mathrm{el}$ y $10 \mathrm{el}$, respectivamente. Los datos presentados en la Tabla 1 se refieren a las estadísticas de la muestra de referencia y las pequeñas muestras que se utilizan. Cabe señalar que se realizaron otras pruebas con pequeñas muestras, teniendo resultados similares a los presentados en este trabajo. 
Tabla 1: Estadísticas de la muestra de referencia y de las pequeñas muestras $\left[\mathrm{cm}^{3} / \mathrm{Hh}\right]$

\begin{tabular}{|l|c|c|c|c|}
\hline Estadística & Muestra 160el & Muestra 20el & Muestra 15el & Muestra 10el \\
\hline Máximo & 130.30 & 75.90 & 88.90 & 130.30 \\
\hline Mínimo & 2.40 & 7.00 & 8.00 & 8.00 \\
\hline Média & 26.14 & 23.80 & 26.79 & 38.99 \\
\hline D. Estándar & 18.81 & 18.98 & 22.04 & 37.89 \\
\hline Mediana & 20.71 & 15.80 & 16.70 & 34.65 \\
\hline Moda & 8.00 & 7.00 & 8.00 & 8.00 \\
\hline Coef. Variación & 0.72 & 0.80 & 0.82 & 0.97 \\
\hline
\end{tabular}

Función de generación y de las clases de los histogramas

Según Morano (2003), Zio et. al. (2006), Tipper (2008), Wu (2008), Constancio (2009), la función de generación para la simulación de Monte Carlo se define utilizando la curva del tipo Beta. Por otra parte, Gupta et al. (2008), Royall (2000), Triola (1999), Nascimento et al. (2009) y Batista et al. (2009), consideran el método de la máxima verosimilitud para la comparación de las funciones Beta y Weibull. Estas funciones son de distribución continua de probabilidad, adonde la función Beta varía entre 0 y 1 y la función Weibull entre 0 y $+\infty$. La función generadora para las muestras de $160 \mathrm{el}, 20 \mathrm{el}$, 15el y $10 \mathrm{el}$, presentan los parámetros de forma y de escala de las funciones mostradas en la Tabla 2.

Tabla 2: Parámetros de forma y de escala de las funciones Beta y Weibull

\begin{tabular}{|c|c|c|c|c|}
\hline Muestra & \multicolumn{2}{|c|}{ Función Beta } & \multicolumn{2}{c|}{ Función Weibull } \\
\hline & Alfa & Beta & $\mathrm{a}$ & $\mathrm{b}$ \\
\hline $160 \mathrm{el}$ & 2.46 & 91.36 & 29.32 & 1.55 \\
\hline $20 \mathrm{el}$ & 1.48 & 4.46 & 26.50 & 1.44 \\
\hline $15 \mathrm{el}$ & 1.24 & 2.99 & 29.82 & 1.42 \\
\hline $10 \mathrm{el}$ & 1.37 & 33.74 & 30.20 & 1.79 \\
\hline
\end{tabular}

Con respecto al criterio de máxima verosimilitud, se llevó a cabo una evaluación de cada muestra con la distribución Beta y Weibull, adoptando un nivel de significancia estadística del 99\%. Esta evaluación tiene el objetivo de identificar la función que mejor se adapte a la forma de la distribución de los datos. La máxima verosimilitud relativa es obtenida por el cálculo del menor valor algébrico de la máxima log- verosimilitud de las funciones en que cada muestra es sometida, dividido por el número de elementos de la muestra, para el caso de la función Beta. En el caso de la función Weibull, la misma es representada por el mayor valor algébrico de la máxima logverosimilitud calculado para cada muestra, dividida por el número de elementos da la muestra; en conformidad con lo establecido por el software MathWorks (2007). Los resultados de la máxima verosimilitud relativa fueron calculados y son presentados en la Tabla 3.

Tabla 3: Máxima Verosimilitud Relativa de las muestras del proceso de soldadura

\begin{tabular}{|l|c|c|c|c|}
\hline Função\Amostra & $160 \mathrm{el}$ & $20 \mathrm{el}$ & $15 \mathrm{el}$ & $10 \mathrm{el}$ \\
\hline Beta & -2.77 & -0.51 & -0.33 & -2.28 \\
\hline Weibull & 4.17 & 4.07 & 4.19 & 4.64 \\
\hline
\end{tabular}

Todas las muestras analizadas por la función Weibull mostraron valores más altos de máxima verosimilitud. De esta manera, la función Weibull es ejemplo de mejor representatividad del proceso de soldadura. Como la muestra de referencia es conocida, es posible comparar y afirmar que la pequeña muestra que mejor representa el proceso es $15 \mathrm{el}$, ya que el valor de su máxima verosimilitud es el más cercano al de la muestra 160el. En el caso de la muestra de referencia al no ser conocida, se identifica que la muestra con la mayor verosimilitud es la muestra $10 \mathrm{el}$. 
Para la definición de las clases y la preparación de los histogramas, se utiliza el criterio de "Sturges", Morano (2003), y se establece la cantidad de números pseudo-aleatorios utilizando las recomendaciones de Cochran (1954), Flanagan (1993) y Morano (2003). Por lo tanto, con las clases definidas y su valor medio, es posible visualizar las FDP y FPA de cada muestra y, enseguida, se puede iniciar el proceso de simulación. En el método de simulación de LHS, se divide la muestra en estratos y se considera la cantidad de elementos de cada estrato. Estos elementos representan una proporción a la muestra total y, cada estrato es representado por su media, su desviación estándar, máximo y mínimo, siendo establecida una función generatriz para cada estrato. En este trabajo se utilizó la distribución Normal. Para establecer el número de estratos se utilizó el mismo criterio para la definición de clases que la empleada en el MMC, así como la cantidad de números pseudo-aleatorios que serán generados y distribuidos en proporción al número de elementos en cada estrato del análisis de la muestra.

\section{Simulaciones de las Muestras Pequeñas}

Con la FDP, el nivel de significancia, el número de clases y la cantidad de números pseudo aleatorios son establecidos para ejecutar la simulación de MMC. Por lo tanto, se generan los números pseudo aleatorios distribuidos en las clases y se identifica la FDP y FPA de la simulación. A continuación, debe realizarse una prueba de adherencia de los datos de simulación con los datos de la muestra. Las pruebas son por Chi-cuadrado y de la máxima verosimilitud en la simulación y en la muestra. Aprobada la adherencia de la simulación en la muestra se calcula a estadística de la simulación y se realiza la construcción de las figuras con los resultados obtenidos. Para la estructuración del modelo de simulación con el método de LHS, las muestras se dividen en estratos, de manera semejante al utilizado en el método de Monte Carlo.

Esta estructuración consiste en la siguiente secuencia: 1) En la muestra de cada estrato se calcula el valor medio, la cantidad de elementos, el promedio y el desvío estándar de estos valores. En los estratos que por acaso no contenga por lo menos un elemento de la muestra, el promedio y el desvío estándar se consideran como el valor medio del estrato y cero, respectivamente. Siendo posible, esta condición debe ser evitada mediante la reducción del número de estratos. 2) Con el número de elementos en cada estrato se calcula el porcentaje correspondiente, por lo que es posible visualizar la FDP y FPA en la muestra. 3) El porcentaje de elementos pertenecientes a cada estrato proporciona la distribución porcentual de números pseudo aleatorios que pertenecen a cada estrato. La forma de la distribución utilizada para generar estos números es del tipo Normal y se estructura según el software MathWorks (2007). 4) En este paso se comienza la simulación para la generación de los números pseudo aleatorios para cada estrato. Luego, se identifican la FDP y la FPA de la simulación efectuada. 5) A continuación, es necesario llevar a cabo una prueba de adherencia de los datos de la simulación con los datos de la muestra. Las pruebas realizadas son el de Chi-cuadrado y de la máxima verosimilitud, tanto en la simulación como en la muestra. 6) Aprobada la adherencia de la simulación en relación a la muestra se calcula la inferencia estadística y se realiza la construcción de las figuras con los resultados obtenidos.

\section{RESULTADOS}

Los resultados obtenidos del análisis de las muestras pequeñas 20el, 15el y $10 \mathrm{el}$, así como la muestra de la referencia $160 \mathrm{el}$ se presentan a continuación. En primer lugar, se identifica la forma de la distribución con el análisis de la muestra 160el. Por lo tanto, en la Fig. 1 se observa la representación de la FDP y FPA de la muestra 160el.

Esta muestra de referencia retrata la característica del proceso en cuestión. Las simulaciones de las muestras más pequeñas deben presentar un comportamiento similar a la muestra de referencia. En la Fig. $1 \mathrm{~b}$ se observa que la productividad para el $100 \%$ del proceso de soldadura es de $120 \mathrm{~cm}^{3} / \mathrm{Hh}$. La productividad para $95 \%$ y $90 \%$ son, respectivamente, 55 y $40 \mathrm{~cm}^{3} / \mathrm{Hh}$. Por lo tanto, se obtiene una referencia básica para la productividad del proceso, esto es a fin de obtener un dimensionamiento más adecuado acerca de la mano de obra utilizada. 
En la simulación por el MMC se evaluó inicialmente la FDP que mejor se ajusta a la muestra. Las funciones consideradas fueron evaluadas por medio de la máxima verosimilitud. En este caso, la Weibull fue la función que mejor se ajusta para todas las pequeñas muestras.

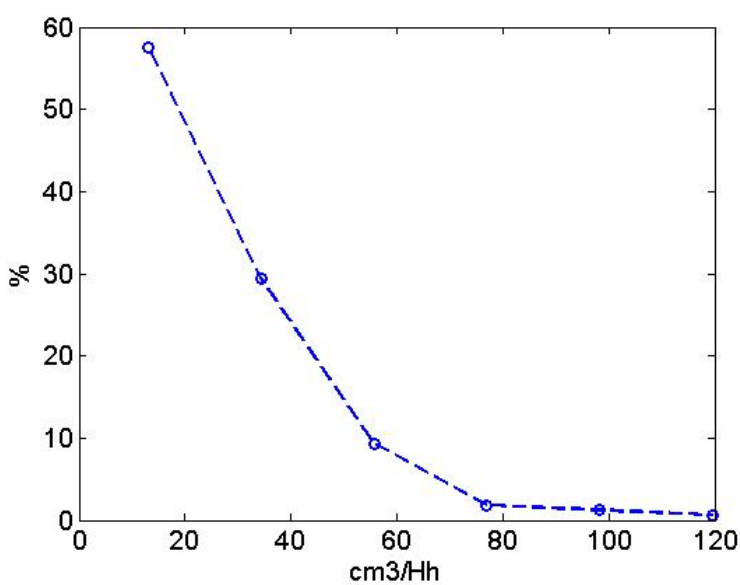

a) FDP de la muestra $160 \mathrm{el}$

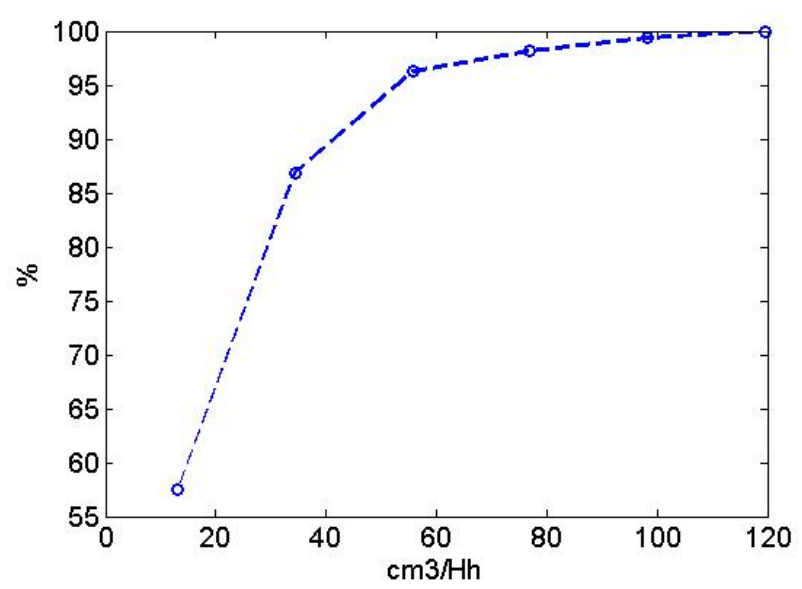

b) FPA de la muestra $160 \mathrm{el}$

Fig. 1: FDP y FPA de la muestra 160el.

Efectuando la simulación por el MMC, utilizando a FDP de Weibull y admitiendo un nivel significancia de 99\%, con una cantidad de 1000 números pseudo aleatorios se obtiene la estadística representada en las Tablas 4, 5 y 6 para la simulación de $20 \mathrm{el}$, 15el y 10el, respectivamente. De una manera similar, la simulación por el método LHS, con los números pseudo aleatorios divididos proporcionalmente al porcentaje atribuido a la distribución de elementos de la muestra entre los estratos, se obtiene la estadística presentada en las Tablas 4, 5 y 6 para la simulación de $20 \mathrm{el}, 15 \mathrm{el}$ y $10 \mathrm{el}$, respectivamente.

Tabla 4: Estadística de la Simulación de $20 \mathrm{el}\left[\mathrm{cm}^{3} / \mathrm{Hh}\right]$

\begin{tabular}{|l|c|c|c|c|}
\hline Estadística & Muestra & MMC Beta & MMC Weibull & LHS \\
\hline Máximo & 75.90 & 75.90 & 75.90 & 75.90 \\
\hline Mínimo & 7.00 & 7.13 & 7.05 & 7.95 \\
\hline Media & 23.80 & 27.62 & 22.22 & 23.75 \\
\hline D. Estándar & 18.98 & 14.00 & 10.98 & 18.54 \\
\hline Mediana & 15.80 & 24.93 & 19.84 & 15.93 \\
\hline Moda & 7.00 & 7.13 & 7.04 & 57.90 \\
\hline Coef. Variación & 0.80 & 0.51 & 0.49 & 0.78 \\
\hline Esperanza & 24.06 & 27.82 & 22.31 & 23.99 \\
\hline
\end{tabular}

Tabla 5: Estadística de la Simulación de $15 \mathrm{el}\left[\mathrm{cm}^{3} / \mathrm{Hh}\right]$

\begin{tabular}{|l|c|c|c|c|}
\hline Estadística & Muestra & MMC Beta & MMC Weibull & LHS \\
\hline Máximo & 88.90 & 88.90 & 88.90 & 88.90 \\
\hline Mínimo & 8.00 & 8.21 & 8.23 & 2.60 \\
\hline Media & 26.79 & 33.20 & 28.04 & 26.83 \\
\hline D. Estándar & 22.04 & 17.21 & 13.60 & 21.36 \\
\hline Mediana & 16.70 & 29.96 & 25.40 & 17.14 \\
\hline Moda & 8.00 & 8.21 & 8.23 & 88.90 \\
\hline Coef. Variación & 0.82 & 0.52 & 0.49 & 0.80 \\
\hline Esperanza & 27.11 & 33.57 & 28.15 & 27.11 \\
\hline
\end{tabular}


Tabla 6: Estadística de la Simulación de $10 \mathrm{el}\left[\mathrm{cm}^{3} / \mathrm{Hh}\right]$

\begin{tabular}{|l|c|c|c|c|}
\hline Estadística & Muestra & MMC Beta & MMC Weibull & LHS \\
\hline Máximo & 130.30 & 130.30 & 130.3 & 130.30 \\
\hline Mínimo & 8.00 & 8.12 & 8.12 & 9.10 \\
\hline Media & 38.99 & 30.73 & 32.43 & 39.04 \\
\hline D. Estándar & 37.89 & 18.71 & 19.75 & 36.01 \\
\hline Mediana & 34.65 & 26.36 & 27.73 & 34.57 \\
\hline Moda & 8.00 & 8.12 & 8.16 & 73.60 \\
\hline Coef. Variación & 0.97 & 0.61 & 0.61 & 0.92 \\
\hline Esperanza & 39.21 & 32.46 & 39.21 & 39.17 \\
\hline
\end{tabular}

En el MMC las simulaciones fueron consideradas válidas como consecuencia de que: 1) El número mínimo de elementos en cada clase sean mayores que el valor unitario; 2) Las pruebas por Chi-cuadrado están abajo del valor máximo admitido; 3) Las pruebas de verosimilitud relativa de las simulaciones sean idénticas a las de la muestra.

La representación de la FPA de la muestra de referencia y la simulación de MMC y LHS para 20el se muestran en la Fig. 2. Observando la Fig. 2a, se puede afirmar por la aplicación del MMC, con un nivel de significancia del $99 \%$, que la productividad alcanza el valor de $70 \mathrm{~cm}^{3} / \mathrm{Hh}$ para una cota de $100 \%$. Por otra parte, la productividad de las cotas con $95 \%$ y $90 \%$ son, respectivamente, 43 y $35 \mathrm{~cm}^{3} / \mathrm{Hh}$ con el uso de la función de Weibull. Del mismo modo, en la Fig. $2 \mathrm{~b}$, se puede afirmar que en la simulación de la productividad con LHS en la cota $100 \%$ es de $70 \mathrm{~cm}^{3} / \mathrm{Hh}$ y en las cotas de $95 \%$ y $90 \%$ son 58 y $48 \mathrm{~cm}^{3} / \mathrm{Hh}$, respectivamente. De esta manera se configuran los escenarios resultantes de esta simulación.

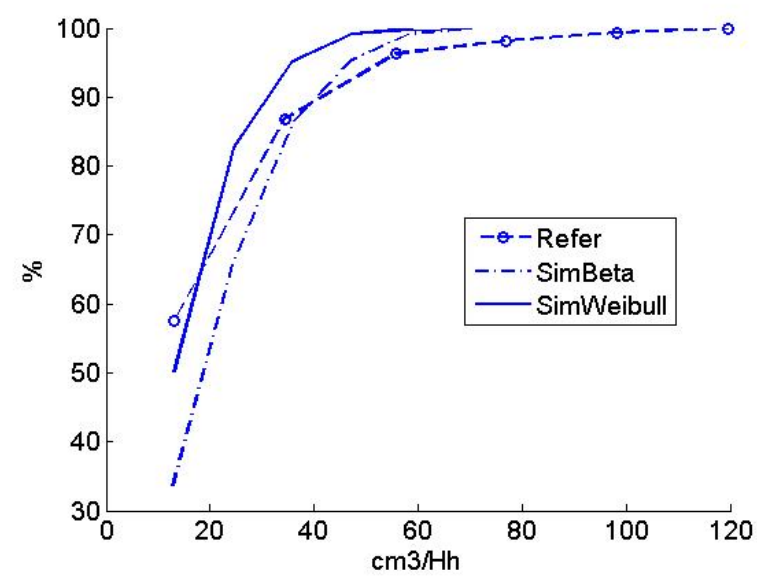

a) FPA de las Simulaciones MMC

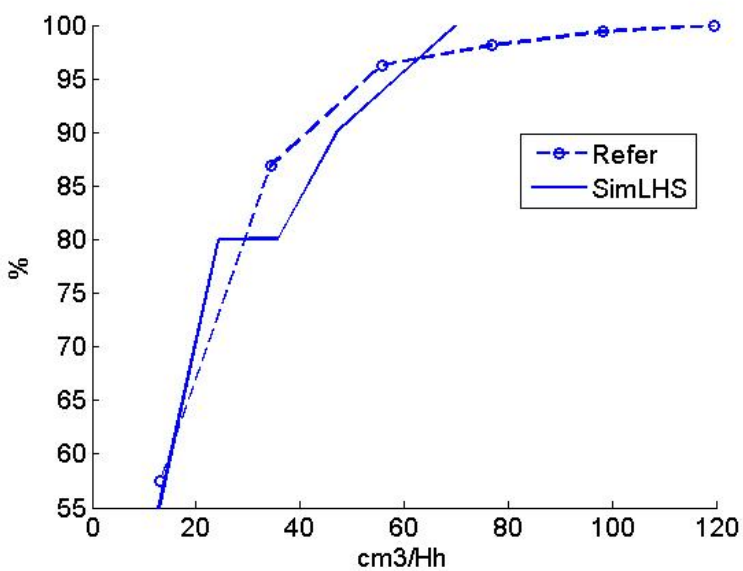

b) FPA de la Simulación LHS

Fig. 2: FPA de la muestra de referencia y simulación de la muestra 20el

La representación de la FPA de la muestra de referencia y la simulación por los métodos de MMC y LHS para 15el se muestran en la Fig 3. Observando la Fig 3a, se puede afirmar por la aplicación del MMC, con un nivel de significancia de $99 \%$, que la productividad alcanza el valor de $80 \mathrm{~cm}^{3} / \mathrm{Hh}$ para una cota de $100 \%$. Por otra parte, la productividad de las cotas con $95 \%$ y $90 \%$ son, respectivamente, 50 y $38 \mathrm{~cm}^{3} / \mathrm{Hh}$ con el uso de la función de Weibull. Del mismo modo, en la Fig. $3 \mathrm{~b}$, se puede afirmar que en la simulación de la productividad con LHS en la cota $100 \%$ es de 80 $\mathrm{cm}^{3} / \mathrm{Hh}$ y en las cotas de $95 \%$ y $90 \%$ son 65 y $45 \mathrm{~cm}^{3} / \mathrm{Hh}$, respectivamente. De esta manera se configuran los escenarios resultantes de esta simulación.

La representación de la FPA de la muestra de referencia y la simulación de MMC y LHS para 10el se muestran en la Fig. 4. Observando la Fig. 4a, se puede afirmar por la aplicación del MMC, con un nivel de significancia de $99 \%$, que la productividad alcanza el valor de $120 \mathrm{~cm}^{3} / \mathrm{Hh}$ para una 
cota de $100 \%$. Por otra parte, la productividad de las cotas con $95 \%$ y $90 \%$ son, respectivamente, 50 y $42 \mathrm{~cm}^{3} / \mathrm{Hh}$ con el uso de la función de Weibull. Del mismo modo, en la Fig. $4 \mathrm{~b}$, se puede afirmar que en la simulación de la productividad con LHS en la cota $100 \%$ es de $120 \mathrm{~cm}^{3} / \mathrm{Hh}$, así como en las cotas de $95 \%$ y $90 \%$ son 110 y $70 \mathrm{~cm}^{3} / \mathrm{Hh}$, respectivamente. De esta manera se configuran los escenarios resultantes de esta simulación

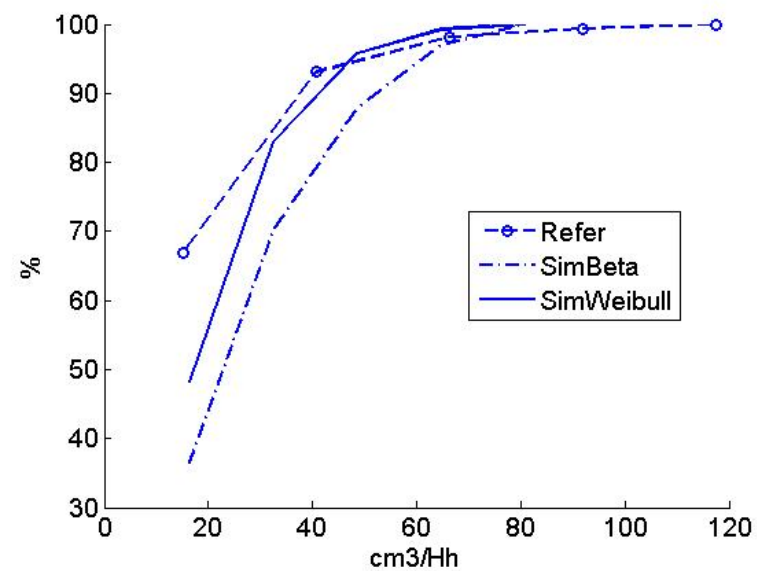

a) FPA de las Simulaciones MMC

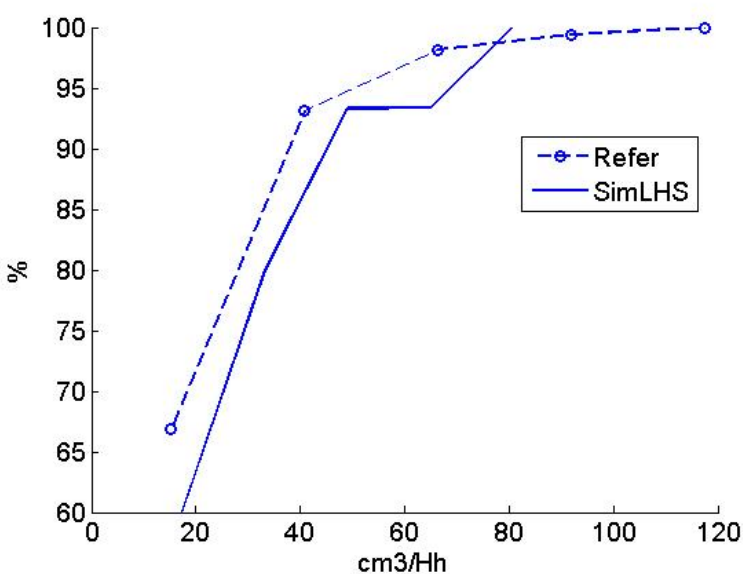

b) FPA de la Simulación LHS

Fig. 3: FPA de la muestra de referencia y simulación de la muestra 15el

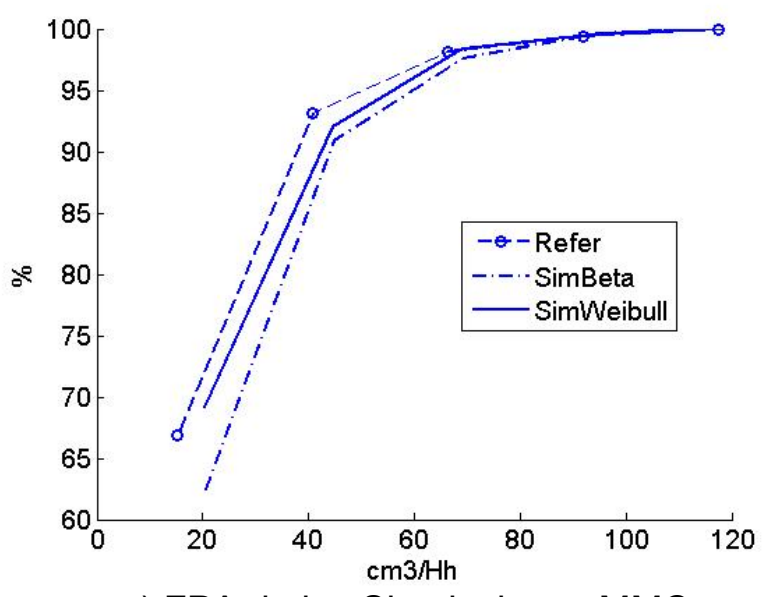

a) FPA de las Simulaciones MMC

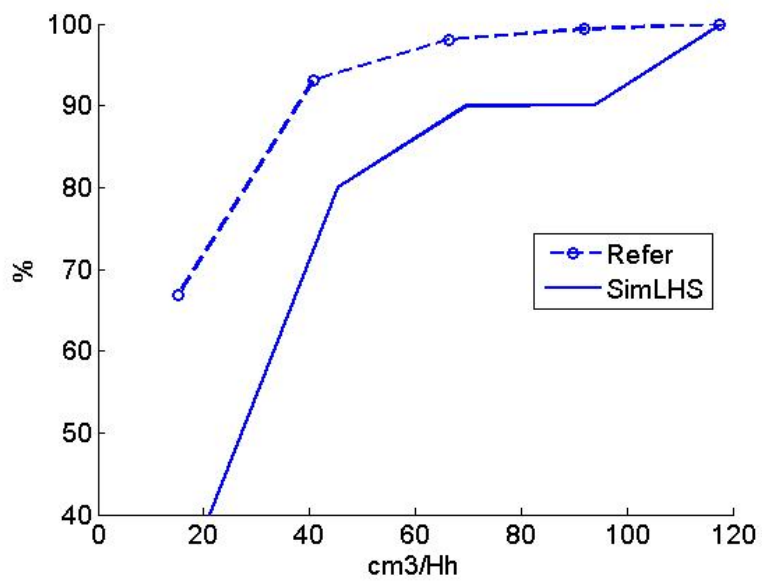

b) FPA de la Simulación LHS

Fig. 4: FPA de la muestra de referencia y simulación de la muestra 10el

\section{DISCUSIÓN DE LOS RESULTADOS}

Observando los resultados presentados por las simulaciones de MMC y LHS puede afirmarse que ambos métodos tienen la misma capacidad de estimar la productividad del proceso de soldadura eléctrica por electrodo revestido con muestras pequeñas. En los casos analizados, las funciones obtenidas por las simulaciones son similares a la muestra de referencia. Las observaciones que se identifican pueden resumirse en los valores de la media y desviación estándar del proceso, siendo respectivamente 26.14 y $18.81 \mathrm{~cm}^{3} / \mathrm{Hh}$, para la muestra de referencia 160el (ver Tabla 1).

La evaluación de la función de máxima verosimilitud aplicable al análisis permite la comparación de la función que mejor se adapte a la distribución de los elementos de las muestras analizadas por el MMC. El análisis de la productividad mostró que la función que mejor se identifica al proceso característico es la distribución de Weibull, como lo demuestran las gráficas de la FPA de las simulaciones en comparación con la muestra de referencia 160el (ver Figuras 2a, 3a y 4a).

Con respecto a la cantidad de números pseudo-aleatorios generados en la simulación de cada muestra se utilizó el máximo de 1000 números teniéndose el cuidado de verificar la cantidad 
mínima de un elemento por clase en el MMC. Por lo tanto, no hubo necesidad de alterar el número de clases calculadas por la regla de Sturges.

El valor de la productividad máxima esperada, leída en la cota del $100 \%$ de la FPA, es un valor bastante interesante, ya que requiere una menor cantidad de $\mathrm{Hh}$ para realizar la tarea. Por lo tanto, al evaluar la productividad de un proceso de soldadura en $\left[\mathrm{cm}^{3} / \mathrm{Hh}\right.$ ] la FPA calculada representa la productividad posible del proceso para un valor porcentual mínimo, pasando por el valor medio, mediana, moda, no necesariamente en este orden, hasta el valor porcentual del $100 \%$ que corresponde a la productividad máxima esperada. Para la evaluación de la capacidad en la cota del $100 \%$ y tomando como ejemplo la cota del $95 \%$ se puede observar el estado de cero variabilidades de la tangente en la curva de la FPA, siendo el máximo alcanzable por el proceso en análisis. Por lo tanto, debe tenerse en cuenta en la productividad de las probabilidades del $100 \%, 95 \%, 90 \%$, el valor medio y mínimo para evaluar la variación entre estos valores. La comparación de la tangente en cada punto de la FPA representa la inclinación de esta función. Cuanto menor sea la tangente, mayor será el cambio de la productividad en cuestión, que resultará en una mayor dispersión. De esta manera observando las Figs. 2, 3 y 4 se puede analizar, de forma cualitativa, que la mejor muestra que se compara con la muestra de referencia es la $15 \mathrm{el}$. Las muestras que mejor se adaptaron a las simulaciones son las $20 \mathrm{el}$ y $15 \mathrm{el}$. Se observa que el método LHS es fuertemente influenciado por una muestra representativa, ya que la FPA de las simulaciones sigue la configuración de estas muestras. Tal como se ha comentado, mediante la aplicación del MMC, diversos trabajos indican que las muestras con la función Beta son las que mejor se adaptan. Mientras que, en este trabajo la función Weibull fue la función que tuvo un mejor ajuste.

Las estimaciones calculadas para el control de la productividad son la mejor forma de definir la capacidad de identificar la estimación de la productividad optimista y pesimista, así como el valor medio, como se observa en la Tabla 7 , donde se presentan los escenarios de los ejemplos utilizados en este trabajo.

Tabla 7: Escenarios de Productividad de los Ejemplos [ $\left.\mathrm{cm}^{3} / \mathrm{Hh}\right]$

\begin{tabular}{|l|c|c|c|}
\hline \multicolumn{1}{|c|}{ Muestra } & Optimista (90\% FPA) & Productividad Media & Pesimista (mínimo) \\
\hline 160el (Referencia) & 42.00 & 26.14 & 2.40 \\
\hline MMC 20el & 37.00 & 22.22 & 7.05 \\
\hline MMC 15el & 35.00 & 28.04 & 8.23 \\
\hline MMC 10el & 41.00 & 32.43 & 8.12 \\
\hline LHS 20el & 48.00 & 23.75 & 7.95 \\
\hline LHS 15el & 45.00 & 26.83 & 8.75 \\
\hline LHS 10el & 70.00 & 39.04 & 9.10 \\
\hline
\end{tabular}

Por lo tanto, escogiendo criterios para el uso de la metodología presentada es posible considerar que las evaluaciones de la productividad son para una perspectiva optimista, de alrededor de la cota $90 \%$, pues presenta una menor dispersión en relación a la cota del $100 \%$; no obstante, para una perspectiva media fue adoptada la cota del valor medio y finalmente para una perspectiva pesimista fue considerada la cota del valor mínimo reportado por cada muestra pequeña. Este hecho es de importancia fundamental para el control de la productividad de una empresa durante operaciones con soldadura eléctrica, ya que permite un control periódico en cortos intervalos de tiempo y así promover correcciones eventuales de rumbo, con el fin de garantizar el cumplimiento de plazos y presupuesto de la obra.

\section{CONCLUSIONES}

En los resultados presentados, se comprobó la aplicabilidad de la simulación por métodos de Monte Carlo e Hipercubo Latino en la estimación de la productividad del proceso de soldadura eléctrica por electrodo revestido en tuberías de la industria brasilera, con base en muestras 
pequeñas. Los resultados muestran que es posible considerar cual tipo de estrategia que se debe utilizar para los valores adoptados en la licitación, elaboración de presupuestos y en la estimación de plazos en obras de construcción y montaje. La definición de esta estrategia podría ser sustancialmente diferente utilizando los valores de la productividad referidos en este trabajo. En este sentido, la toma de decisión sobre cual indicador de productividad se debe utilizar para considerar estos escenarios, es una práctica que no es utilizada hoy en día por la industria.

\section{REFERENCIAS}

AWS - American Welding Society. Welding - Related Expenditures and Productivity Measurement in U.S. Manufacturing, Construction, and Mining Industries. 2002. 90 p.

Batista J. L. F. y H. T. Z. Couto, A razão de verossimilhança como alternativa aos testes de aderência: um exemplo de distribuição diamétricas de florestas tropicais, 47a. Reunião Anual da Região Brasileira da Sociedade Internacional de Biometria (RBRAS), Rio Claro - Programa e Resumos, 42-46, (2002).

Brito J. D. y R. Paranhos, Como Determinar os Custos da Soldagem, Ed. Paranhos, (2005).

Cochran W. G., The Combination of Estimates from Different Experiment,. Biometrics, 10, 1, 101129, (1954)

Constâncio D. S., M. L. R. Ferreira, y I. J. Freire, Estimativa de Produtividade na Soldagem de Tubulações Industriais utilizando o Método de Monte Carlo. XXXV CONSOLDA - Congresso Nacional de Soldagem; ABS - Associação Brasileira de Soldagem. Piracicaba, São Paulo, Brasil, (2009).

Diekmann J. E. y J. Heinz, Determinants of jobsite productivity, Construction Industry Institute Research Rep. No. 143-11, Univ. of Texas at Austin, (2001).

Flanagan R.y G. NORMAN, Risk Management and Construction, London, Blackwell Science, 208 p, (2003).

Gioia A. L. S. ; I. F. da Silva Junior, M. L. R. Ferreira, D. P. Lannes, M. P. Maia, J. Pardal, Avaliação da Metodologia da Produtividade na Atividade de Montagem de Tubulações em Obras Industriais, $10^{\circ}$ COTEQ - ABENDE, Salvador, (2009).

Gupta A., B. Mukherjee y S. K. Upadhyay, Weibull extension model: A Bayes study using Markov chain Monte Carlo simulation, Department of Statistics, \& DST Centre for Interdisciplinary Mathematical Sciences, Banaras Hindu University, India, Reliability Engineering and System Safety 93, 1434-1443, (2008).

Martins, J. L. F. y M. L. R. Ferreira, Avaliação da Produtividade do Processo de Soldagem de Eletrodo Revestido através de Simulação pelo Método do Hipercubo Latino, X Congresso IberoAmericano em Engenharia Mecânica - CIBEM 10, 3579 - 3588, Porto, Portugal, 4 al 7 de Setembro (2011).

Martins J. L. F., M. L. R. Ferreira y J. M. F. Saraiva, Estimativa da Produtividade em Soldagem pelo Método de Monte Carlo, Soldagem \& Inspeção, 16, 03, 204-212, (2011).

Maschio, C.; C. P. V. Carvalho y D. J. Schiozer, Aplicação da Técnica do Hipercubo Latino na Integração do Ajuste de Histórico com a Análise de Incertezas, $5^{\circ}$ Congresso Brasileiro de Pesquisa e Desenvolvimento em Petróleo e Gás, Associação Brasileira de P\&D em Petróleo e Gás - ABPG, UFC, Fortaleza-CE, Outubro (2009).

MathWorks, MATLAB, The language of technical computing. Using MATLAB, Ver.6. The MathWorks Inc. Natick, MA, USA, (2001). 
MathWorks, Statistics Toolbox ${ }^{\mathrm{TM}}$ 6. User's Guide, The MathWorks Inc, (2007).

Morano, C. A. R. y M. L. R. Ferreira, Aplicação do Método de Monte Carlo em Análise de Riscos em Projetos de Construção,. XXXV SBPO - Simpósio Brasileiro de Pesquisa Operacional, Natal, (2003).

Morano C. A. R. y M. L. R. Ferreira, Metodologias de Gerenciamento de Risco em Projetos, Engevista (UFF), 53 - 65, Niterói, Rio de Janeiro, Brasil., 10 jun (2003).

Nascimento L.S.V., J. N. B. Campos y T. M. C. Studart, Análise da Eficiência dos Métodos dos Momentos e da Máxima Verossimilhança na Estimativa de Parâmetros da Distribuição Gama II: Uma Abordagem Probabilística, XV Simpósio Brasileiro de Recursos Hídricos, Curitiba, Porto Alegre, (2003).

Page J. S. y J. G. Nation, Estimator's Piping Man Hour Manual, First Revision, Gulf Pub Comp. Houston, Texas, (1967).

Pilger G. G, J. F. C. L. Costa y J. C Koppe, Improving the Efficiency of the Sequential Simulation Algorithm Using Latin Hypercube Sampling, Geostatistics Banff 2004, 14, Springer, 989-998, (2005).

PROMINP (Programa de Mobilização da Indústria do Petróleo e Gás Natural). Projeto E\&P 27.5 Métricas de Desempenho da Indústria Nacional, Padrões de Métricas da Indústria EPC Nacional, (2010).

Royall R.M, Statistical evidence: a likelihood paradigm. Boca Ranton: Chapman \& Hall/CRC, 1997, $191 \mathrm{p}$.

Tipper J. C, A simple method for representing some univariate frequency distributions, with particular application in Monte Carlo-based simulation, Computers \& Geosciences, 34, (2008).

TRIOLA, Mário F. Introdução à Estatística. 7a. Ed. Rio de Janeiro: LTC, (1999).

Wu Yun-Fu, Correlated sampling techniques used in Monte Carlo simulation for risk assessment, Nuclear Safety Department, Taiwan Power Company, 242, Roosevelt Road, Section 3, Taipei, Taiwan 100, Republic of China, International Journal of Pressure Vessels and Piping, 662-669, (2008).

Zio E.; P. Baraldi y E. Patelli, Assessment of the availability of an offshore installation by Monte Carlo simulation, Dipartimento Ingegneria Nucleare, Politecnico di Milano, Milan, Italy, International Journal of Pressure Vessels and Piping 83, 312-320, (2006). 